\title{
A chromosomal study on a Lebanese spittlebug Philaenus arslani (Hemiptera: Auchenorrhyncha: Aphrophoridae)
}

\author{
ANNA MARYAŃSKA-NADACHOWSKA ${ }^{1}$, VALENTINA G. KUZNETSOVA² and HANI ABDUL-NOUR ${ }^{3}$ \\ ${ }^{1}$ Department of Experimental Zoology, Institute of Systematics and Evolution of Animals, Polish Academy of Sciences, \\ Sławkowska 17, 31-016 Kraków, Poland; e-mail: maryanska@isez.pan.krakow.pl \\ ${ }^{2}$ Department of Karyosystematics, Zoological Institute, Russian Academy of Sciences, 199034 St. Petersburg, Russia; \\ e-mail: karyo@zin.ru
}

${ }^{3}$ B.P. 90 549, Jdeidet al-Matn, 12022050 Lebanon, e-mail: hani-abd@inco.com.lb

Key words. Hemiptera, Auchenorrhyncha, Aphrophoridae, Philaenus arslani, chromosomes, C-bands, DAPI, CMA 3 , NOR

\begin{abstract}
The meadow spittlebug genus Philaenus (Auchenorrhyncha: Aphrophoridae) is known to display marked colour polymorphism. This study presents the results of a karyotype analysis of $P$. arslani from Lebanon using conventional chromosome staining, C-banding, fluorescent banding using base-specific fluorochromes $\left(\mathrm{CMA}_{3}\right.$ and DAPI) and AgNOR-staining. This species has $2 \mathrm{n}=18$ + neo-XY, and differs from P. spumarius both in the number of chromosomes and sex chromosome system. During meiosis, the neo-XY bivalent is clearly heteromorphic being the largest in the complement. Furthermore, sex chromosomes show marked differences in C-banding pattern. The NOR-bearing chromosomes are the first and one of the middle-sized pairs of autosomes. NORs are G-C rich. Furthermore, some blocks of constitutive heterochromatin on the sex chromosomes are also G-C rich. All other C-bands are DAPI or DAPI/ $\mathrm{CMA}_{3}$ positive, thus containing A-T rich DNA. The significant difference in the karyotype of $P$. arslani and $P$. spumarius indicates chromosomal transformations during the evolution of the genus Philaenus.
\end{abstract}

\section{INTRODUCTION}

Until quite recently the genus Philaenus Stål, 1864 was considered to include three species only: the holarctic $P$. spumarius (Linnaeus, 1758), the Mediterranean P. signatus Melichar, 1896 and P. tesselatus Melichar, 1889. The latter, of unclear taxonomic status, is regarded also as a synonym of $P$. spumarius (see Drosopoulos \& Quartau, 2002). Recent studies increased the number of Philaenus species to 8 and all of the newly described species were discovered in the Mediterranean (Drosopoulos \& Asche, 1991; Loukas \& Drosopoulos, 1992; Abdul-Nour \& Lahoud, 1995; Drosopoulos \& Remane, 2000; Remane \& Drosopoulos, 2001; Drosopoulos \& Quartau, 2002). Of these species, P. spumarius, P. tesselatus, P. loukasi Drosopoulos and Asche, 1991 and $P$. arslani comprise the "spumarius" group, whereas $P$. tarifa Remane and Drosopoulos, 2001, P. signatus, $P$. italosignus Drosopoulos and Remane, 2000 and $P$. maghresignus Drosopoulos and Remane, 2000 belong to the "signatus" group, however this division is still ambiguous (Loukas \& Drosopoulos, 1992; Drosopoulos \& Remane, 2000; Drosopoulos, 2003). P. arslani was described from Lebanon, where it occurs in mountains at altitudes between 1000-2000 m. This species is associated with arid plants such as Eryngium, Echinops, Carduus, Cirsium, Cistus, and expresses only one colour morph named populi (Abdul-Nour \& Lahoud, 1995). Quite recently, populations of this species were also found in SE Turkey and in Iraq (see Drosopoulos, 2003).

Up to now the only Philaenus species studied cytogenetically, $P$. spumarius, has $2 \mathrm{n}=22+\mathrm{XX} / \mathrm{X} 0$ (Boring,
1913; Kurokawa, 1953; Kuznetsova et al., 2003). Some additional information on the karyotype of $P$. spumarius was also provided by several methods of differential chromosome staining (C-, AgNOR- and fluorescentbanding) (Kuznetsova et al., 2003).

The genus Philaenus is known to show marked colour polymorphism. The nature and origin of this polymorphism and its possible contribution to the evolution of reproductive isolation and sympatric speciation have been extensively documented for P. spumarius (for an overview of the literature, see Halkka \& Halkka, 1990; Stewart \& Lees, 1996; Drosopoulos, 2003). Alteration of the karyotype may play a substantial role in the process of speciation (King, 1993). Therefore, an interesting question is whether this is the case for the genus Philaenus, which is considered to be one of the best insect genera for evolutionary studies on polymorphism and speciation (Drosopoulos \& Quartau, 2002).

This paper is part of a project exploring the cytogenetics of the genus Philaenus and presents the karyotype of another representative of this group, $P$. arslani, described from Lebanon, which as far as is known does not show colour polymorphism (Abdul-Nour \& Lahoud, 1995).

\section{MATERIAL AND METHODS}

Specimens of $P$. arslani were collected by H. Abdul-Nour in the mountains of Lebanon, in August 2004, from Lassa (Kesrouane), altitude: $1200 \mathrm{~m}$ and in June-July 2005 from Mazraat Kfar Zebiane (Kesrouane), altitude: $1600 \mathrm{~m}$. Adult specimens were fixed in an ethanol : acetic acid fixative $(3: 1,96 \%$ ethanol : glacial acetic acid). Testis structure of 18 males and ovary 

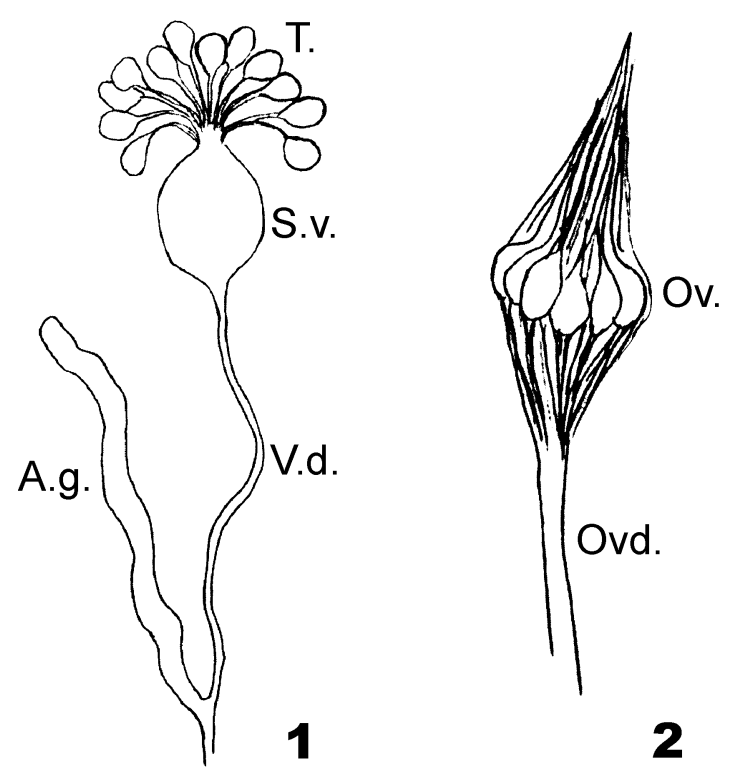

Figs 1-2. Reproductive systems of Philaenus arslani. 1 male; 2 - female. Abbreviations: T. - testis, S.v. - seminal vesicle, V.d. - vas deferens, A.g. - accessory gland, Ov.- ovary, Ovd. - oviduct.

structure of 4 females were studied primarily in terms of the number of testicular follicles and ovarioles. The chromosomal study was based on spermatogenesis in 18 males. Chromosome spread preparations of mitotic and meiotic cells obtained from testicular follicles were made as previously described by Kuznetsova et al. (2003). The standard Feulgen-Giemsa procedure (Grozeva \& Nokkala, 1996) was used for conventional staining. Silver staining of nucleolar organizing regions (NORs) was performed following the technique of Howell \& Black (1980). Some slides were C-banded to visualize constitutive heterochromatin according to Sumner (1972). Chromomycin $A_{3}$ $\left(\mathrm{CMA}_{3}\right)$ and 4'-6'-diamidino-2-phenylindole (DAPI) staining of the C-banded preparations were performed in accordance to Schweizer (1976) and Donlon \& Magenis (1983), respectively. For methodological details of the different staining methods see Kuznetsova et al. (2003). Slides were examined using a Nikon Eclipse E400 fluorescence microscope, at 1000x. The microphotographs were made with a Nikon DS-U1 camera. The images were optimized for best contrast and brightness by means of the Adobe Photoshop 6.0 image-processing software.

\section{RESULTS}

\section{The internal reproductive organs}

In the adult males, the internal reproductive organs comprise (i) a pair of testes, each with $10-11$ or, more often, 12 drop-shaped follicles, (ii) a pair of slender vas deferens, each with a well developed seminal vesicle that is rounded in shape, (iii) the ejaculatory duct, and (iv) a pair of long accessory glands undivided into chambers (Fig. 1). Testes are located dorsally in the proximal part of the abdomen. In the adult females, the paired ovaries lead into paired oviducts, which merge further into a median oviduct. We failed to identify accessory glands. In ovaries, spherical ovarioles are connected to each other at the top by filaments and varied in number between 16 and 19, with no clearly prevailing number in the four females studied (Fig. 2).

\section{Mitotic complement}

Male karyotype of $P$. arslani consists of 20 holokinetic chromosomes. In mitotic complements, a pair of very large chromosomes, one clearly larger than the other, represent sex chromosomes (Figs 3, 4). The larger sex chromosome is probably an $\mathrm{X}$ chromosome and the smaller a $\mathrm{Y}$ chromosome (see Discussion).

\section{Course of meiosis}

Spermatocyte pachytene cells showed two separate positively heteropycnotic bodies of differing size representing the sex chromosomes (Fig. 5). Diplotene, diakinesis and metaphase I revealed 10 bivalents indicative of $2 n=20$ (Figs 6-9). The nine autosomal bivalents gradually decreased in size. Sex chromosome pair was significantly larger than the largest autosomal bivalent and clearly heteromorphic. Each of the bivalents normally formed the only chiasma, however in some cells two or very rarely three chiasmata could be seen in the sex bivalent and in two or even three larger autosomal bivalents. The chiasmata were generally terminally located except for bivalents with three chiasmata, in which one chiasma was interstitial (Figs 6,7). The anaphase I showed two groups of chromosomes, $9 \mathrm{~A}+\mathrm{X}$ and $9 \mathrm{~A}+\mathrm{Y}$ respectively, segregating to opposite poles (Fig. 10). Thus, the male chromosome complement is suggested to be $2 \mathrm{n}=18+\mathrm{XY}$.

\section{C- and fluorescent $\mathrm{CMA}_{3} / \mathrm{DAPI}-b a n d i n g$, AgNOR-staining}

C-banding of mitotic chromosomes revealed small C-bands on the telomeres of autosomes. In contrast to autosomes, C-banding showed prominent heterochromatic regions on both telomeres of the putative $\mathrm{X}$ chromosomes and on one of telomeres of the putative $\mathrm{Y}$ chromosome. On the $\mathrm{X}$ chromosome, one of the bands was about twice as large as the other. On the Y chromosome, telomeric heterochromatin consisted of two closely-spaced bands (Figs 3,4). The banding pattern on the $\mathrm{X}$ and the $\mathrm{Y}$ chromosomes was always visible also in meiosis, independent of their degree of condensation. Telomeric C-bands of the $\mathrm{X}$ chromosome tended to be attracted together, in which case the $\mathrm{X}$ very often formed a ring in mitosis (Fig. 3), whereas in meiotic prophase the $\mathrm{XY}$ bivalent produced an intricate loop (Figs 6, 7). Staining of C-banded chromosomes by fluorochromes revealed that $P$. arslani heterochromatin contained both A-T and G-C rich DNA (Figs 11-15). In meiosis, telomeric heterochromatin of some of autosomes was brightly coloured after DAPI/CMA 3 staining, evidence that A-T and G-C bases are equally represented at this site (Figs $12,13)$. The largest autosome pair and one of the middlesized autosome pairs (6th or 7th) carried only G-C rich heterochromatin indicated by $\mathrm{CMA}_{3}$ bright/DAPI dark sites (Fig. 14, 15). These $\mathrm{CMA}_{3}$ positive sites were argentophilic demonstrating that these chromosomes bear NORs, containing actively transcribed rDNA genes. Furthermore, silver stained nucleolar remnants were seen attached to these chromosomes during meiosis up to diakinesis (Figs 16, 17). 

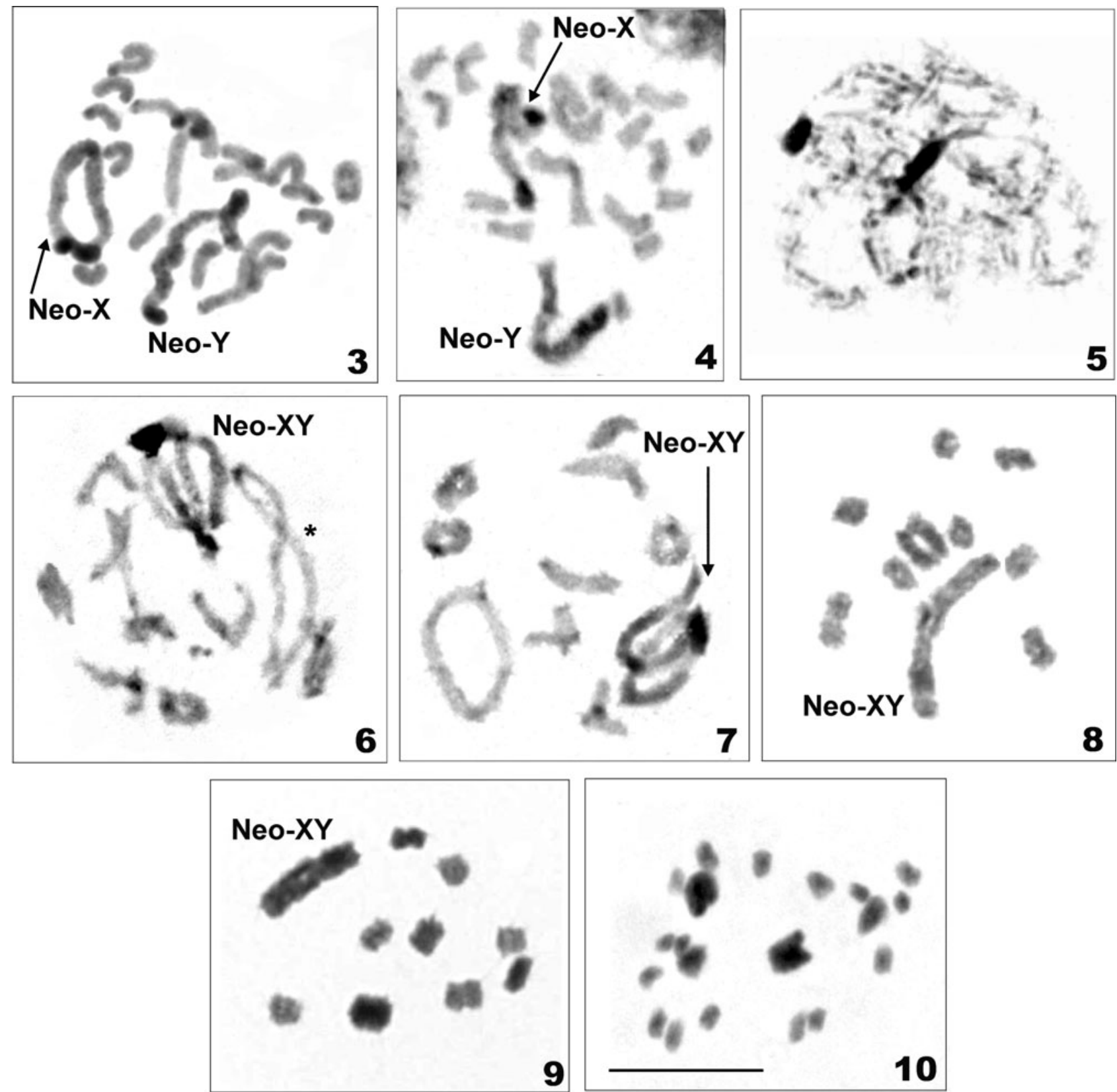

Figs 3-10. Mitotic and meiotic chromosomes of Philaenus arslani males. 3-4: C-banded mitotic chromosomes. 5-10: Meiotic chromosomes stained with Foulgen-Giemsa. 5 - pachytene with two heteropicnotic entities; 6, 7 - diplotene bivalents with one, two (forming rings) and three (star) chiasmata; 8 - diakinesis with large heteromorphic neo-XY bivalent; 9 - metaphase I with large heteromorphic neo-XY bivalent; 10 - anaphase I. Bar indicates $10 \mu \mathrm{m}$.

The telomeric C-band of the Y chromosome and one terminal band of the $\mathrm{X}$ chromosome were brightly fluorescent both after DAPI and $\mathrm{CMA}_{3}$ staining, showing that they are A-T and G-C rich. The other telomeric C-band of the $\mathrm{X}$ chromosome displayed a DAPI bright/CMA $\mathrm{CH}_{3}$ dark fluorescent pattern (Figs 12, 13).

\section{DISCUSSION}

On the basis of the morphology of male and female internal reproductive organs, several characters have been identified, including the number of follicles and ovarioles, which may be useful taxonomically as well in understanding the phylogenetic traits of the Auchenorrhyncha
(Emelyanov \& Kuznetsova, 1983; Kuznetsova, 1985; Kirillova, 1989; Bednarczyk, 1993; Kuznetsova et al., 1998; D'Urso et al., 2005). For the family Aphrophoridae, there is some data on the morphology of testes and ovaries, mainly the number of testicular follicles and ovarioles, for as few as 4 species, including $P$. spumarius (see Emelyanov \& Kuznetsova, 1983; Kuznetsova et al., 2003). These results demonstrate considerable variability in the number of testicular follicles (12-35) and ovarioles (11-20) in the family. Moreover, follicles and ovarioles slightly vary in number between specimens and even between different testes and ovaries of a specimen, as in P. arslani (this study). In this species, between 

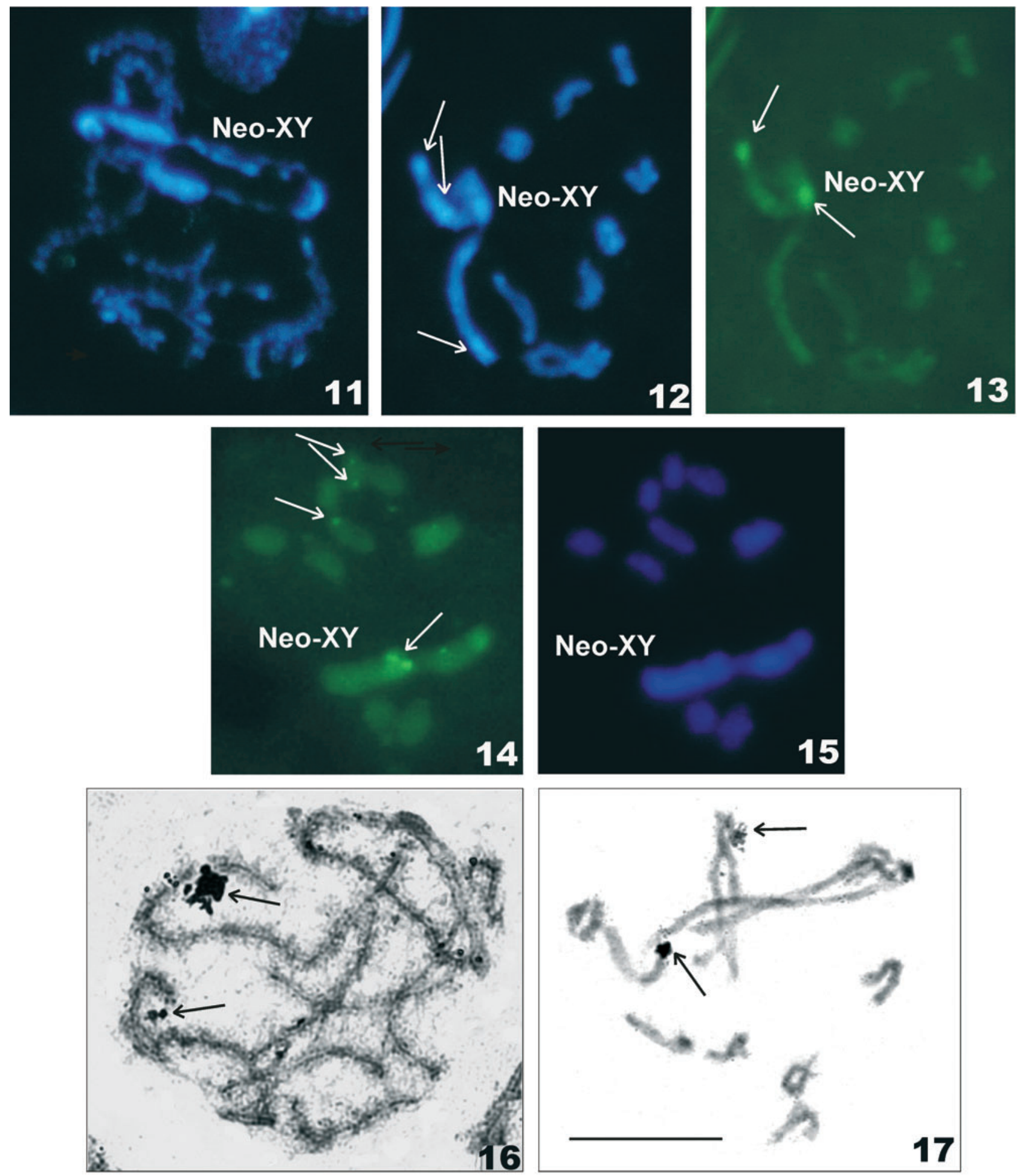

Figs 11-17. Meiotic chromosomes of Philaenus arslani males after DAPI-staining (11, 12, 15), $\mathrm{CMA}_{3}$-staining $(13,14)$ and Agstaining $(16,17) .11$ - diplotene; note DAPI-positive signals on the neo-XY bivalent; 12 - diakinesis; note DAPI-positive bright signals on the neo-XY bivalent (arrows); 13 - diakinesis; note $\mathrm{CMA}_{3}$-positive signals on the neo-XY bivalent (arrows); 14 - metaphase I; note $\mathrm{CMA}_{3}$-positive signals on the neo-XY bivalent and two autosomal bivalents (arrows); 15 - metaphase I without DAPIpositive signals; 16, 17 - diplotene (16) and diakinesis (17), Ag-stained NORs are indicated by arrows. Bar indicates $10 \mu \mathrm{m}$.

10 and 12 follicles and from 16 to 19 ovarioles were found in the 18 males and 4 females studied. In $P$. spumarius, there are 11-13 follicles and 10-12 ovarioles (Ivanov, 1926; Kuznetsova et al., 2003). Hence, congeneric species share a similar testis structure, but differ in the number of ovarioles. However, data for other Philaenus species are required before making inferences.

Previous cytogenetic reports on the family Aphrophoridae, including 22 species from 9 genera, have shown diploid chromosome numbers ranging from 16 to 32 (in 
female) and the occurrence of two sex chromosome determination systems, XX/X0 and XX/XY (see Halkka, 1959; Kirillova, 1986). The representatives of the family, similar to other auchenorrhynchan groups, have holokinetic chromosomes, a chiasmatic pre-reductional male meiosis with predominantly 1-2 chiasmata per bivalent, and fusions/fissions as the basic mode of karyotype evolution, fusions of chromosomes being apparently more common than fissions (Halkka, 1959, 1964; Kuznetsova et al., 2003).

In spite of the high species diversity in the genus Philaenus, only the karyotype of $P$. spumarius has been studied so far. This species has $2 \mathrm{n}=22+\mathrm{XX} / \mathrm{X} 0$ (Boring, 1913; Kurokawa, 1953; Kuznetsova et al., 2003). Furthermore, the karyotype of this species contains two $\mathrm{CMA}_{3}$ positive NORs, associated with the first and one of the middle-sized (6th or 7th) autosome pairs respectively, and very small C-bands located on the telomeres of both autosomes and the $\mathrm{X}$ chromosome (Kuznetsova et al., 2003). The karyotype of P. arslani reported here is distinctly different in chromosome number $(2 \mathrm{n}=18+$ neoXY), sex chromosome system $(\mathrm{XX} / \mathrm{XY})$, and in the greater amount of sex chromosome heterochromatin.

In contrast to the sex chromosomes, autosomes of both species have only dot-like telomeric C- bands. An important point is that $P$. arslani and $P$. spumarius have a similar number and location of NORs, suggesting that the NOR bearing chromosomes were not involved in the rearrangements. The fact that NOR associated heterochromatin stains brightly with $\mathrm{CMA}_{3}$ in both $P$. spumarius and $P$. arslani indicates that it is $\mathrm{G}-\mathrm{C}$ rich. This fluorescence pattern of NOR seems to be a general feature of the hemipteran genome, since it has been described in the great majority of taxa examined so far (Nechaeva et al., 2004; Cattani et al., 2004; Criniti et al., 2005; Lanzone \& De Souza, 2006). Noteworthy is that $P$. spumarius and $P$. arslani differ significantly in the amount and composition of heterochromatin. In P. spumarius, heterochromatin is only found in NOR bearing chromosomes, whereas $P$. arslani displays a considerable amount of heterochromatin, which contains both G-C and A-T rich regions. In this species, heterochromatin in the $\mathrm{X}$ and $\mathrm{Y}$ is both DAPI and $\mathrm{CMA}_{3}$ bright, whereas a telomeric band of the $\mathrm{X}$ is DAPI bright/ $\mathrm{CMA}_{3}$ dark.

The low number of chiasmata seems to be a standard pattern in holokinetic bivalents (Halkka, 1964). Nokkala et al. (2004) analyzed chiasma formation and distribution in holokinetic bivalents of a psyllid species, Beopelma foersteri (Flor) (Sternorrhyncha: Psylloidea). These authors conclude that more than two chiasmata in a holokinetic bivalent must necessarily obstruct the regular course of meiosis and result in the elimination of cells of this sort. In $P$. arslani, two and occasionally three chiasmata were observed in separate bivalents. Very rarely, three and even four chiasmata are likewise found in larger bivalents of P. spumarius (Kuznetsova et al., 2003). The fate of cells with multichiasmatic bivalents remains, however, unknown in both species.
Comparison of karyotypes of both Philaenus species indicates that the XY system of $P$. arslani is formed by neo-sex chromosomes. We suggest that the karyotype of $P$. arslani has been derived from that of $P$. spumarius by means of two fusions, the first between two pairs of autosomes and the second between an autosome and the $\mathrm{X}$ chromosome. As a consequence, the putative neo-Y chromosome and the autosomally derived part of the putative neo-X chromosome of $P$. arslani appear similar in size. They are also similar in that each has a C-band on one of the telomeres. Similar to sex chromosomes with standard kinetic structure (monocentric chromosomes), holokinetic sex chromosomes quite often contain a considerable amount of heterochromatin (Papeschi, 1995; Grozeva \& Nokkala, 2001; Criniti et al., 2005; Kuznetsova et al., 2007), and this is also true for P. arslani. A large C-heterochromatic segment situated on a telomere of the putative $\mathrm{X}$ chromosome consists probably mainly of an original $\mathrm{X}$ chromosome of the ancestor. It is noteworthy that a very similar pattern occurs in Neophilaenus lineatus Linnaeus and $N$. exclamationis Thunberg of the same family. These closely related species have $2 \mathrm{n}=28+$ $\mathrm{XX} / \mathrm{X} 0$ and $2 \mathrm{n}=18+\mathrm{XX} / \mathrm{XY}$, respectively. In $N$. exclamationis, the $\mathrm{X}$ and $\mathrm{Y}$ chromosomes are very large, the $\mathrm{X}$ markedly exceeding the $\mathrm{Y}$ in size and carrying a heterochromatin segment. It is speculated that the heterochromatin segment represents a former $\mathrm{X}$ chromosome (Halkka, 1959). At present, with data available for only two species, little can be inferred about the chromosomal evolution of the genus Philaenus. However, the significant difference in the karyotype of $P$. arslani and $P$. spumarius indicates many chromosomal transformations during the evolution of the genus Philaenus, including structural rearrangements and heterochromatization. We suggest that data for other Philaenus species will reveal further differentiation at the chromosomal and anatomical levels, giving additional insight into the taxonomy and evolution of this speciose genus.

ACKNOWLEDGMENTS. This research was supported by the Ministry of Science and Higher Education, Poland grant no. 303 01731/0639 and in part by grants to V.G. Kuznetsova from the Russian Foundation for Basic Research (no. 05-04-48387) and the Program of the Presidium RAS "Dynamics of Gene Pools in Animals, Plants and Man". We thank two anonymous referees for their comments, which helped greatly to improve this manuscript.

\section{REFERENCES}

Abdul-Nour H. \& Lahoud L. 1995: Révision du genere Philaenus Stål, 1864 au Liban, avec la description d'une nouvelle espèce: P. arslani, n. sp. (Homoptera, Auchenorrhyncha, Cercopidae). Nouv. Rev. Entomol. (N.S.) 12: 297-303.

BEDNARCZYK J. 1993: The structure of male reproductive system in species of the family Cicadellidae (Homoptera, Auchenorrhyncha). Acta Biol. Siles. 22: 126-142.

Boring A.M. 1913: The chromosomes of the Cercopidae. Biol. Bull. 24: 133-147.

Cattani M.V., Graizerstein E.J. \& Papeschi A.G. 2004: Male meiotic behaviour and nucleolus organizer regions in Camptischium clavipes (Fabr.) (Coreidae, Heteroptera) analyses by 
fluorescent banding and in situ hybridization. Caryologia 57: 267-273.

Criniti A., Simonazzi G., Cassanelli S., Ferrari M., Bizzara D. \& MANICARDI G.C. 2005: X-linked heterochromatin distribution in the holocentric chromosomes of the green apple aphid Aphis pomi. Genetica 124: 93-98.

Donlon T.A. \& MAGENIS R.E. 1983: Methyl green is a substitute for distamycin $\mathrm{A}$ in the formation of distamycin A/DAPI C-bands. Hum. Genet. 65: 144-146.

Drosopoulos S. 2003: New data of the nature and origin of colour polymorphism in the spittlebug genus Philaenus (Hemiptera: Aphrophoridae). Ann. Soc. Entomol. Fr. (N. S.) 39: $31-42$.

Drosopoulos S. \& Asche M. 1991: Biosystematic studies on the spittlebug genus Philaenus with the description of a new species. Zool. J. Linn. Soc. 101: 169-177.

Drosopoulos S. \& Quartau J.A. 2002: The spittle bug Philaenus tesselatus Melichar, 1899 (Hemiptera, Auchenorrhyncha, Cercopidae) is a distinct species. Zootaxa 68: 1-8.

Drosopoulos S. \& Remane R. 2000: Biogeographic studies on the spittlebug species group Philaenus signatus with the description of two new allopatric species. Ann. Soc. Entomol. Fr. (N. S.) 36: 269-277.

D'Urso V., Guglielmino A. \& Mauceri A. 2005: The internal male and female reproductive apparatus in Cixidia sikaniae D’Urso \& Guglielmino, 1995 (Fulgoromorpha, Achilidae). Zootaxa 1077: 25-36

Emelyanov A.F. \& Kuznetsova V.G. 1983: The number of seminal follicles as a phylogenetic and taxonomic feature in the Dictyopharidae (Homoptera) and other leafhoppers. Zool. Zh. 62: 1583-1586 [in Russian, English abstr.].

Grozeva S. \& Nokkala S. 1996: Chromosome and their meiotic behaviour in two families of the primitive infraorder Dipsocoromorpha (Heteroptera). Hereditas 125: 31-36.

Grozeva S. \& NokKala S. 2001: Chromosome numbers, sex determining systems, and patters of the C-heterochromatin distribution in 13 species de Lace Bugs (Heteroptera, Tingidae). Folia Biol. (Kraków) 49: 29-41.

Halkka O. 1959: Chromosome studies on the Hemiptera, Homoptera, Auchenorrhyncha. Ann. Acad. Sci. Fen. (A IV) 43: $1-71$.

HalkKa O. 1964: Recombination in six homopterous families. Evolution 18: 81-88.

HalkKa O. \& HalkKa L. 1990: Population genetics of the polymorphic spittlebug, Philaenus spumarius (L.). Evol. Biol. 24: 149-191.

Howell W.M. \& BLACK D.A. 1980: Controlled silver staining of nucleolus organizer regions with a protective colloidal developer: a 1-step method. Experientia 36: 1014-1015.

IvANOv S.P. 1926: Contribution to the knowledge of the sexual apparatus of Homoptera, Cicadoidea. Russ. Entomol. Obozr. 20: 210-227 [in Russian, English abstr.].

KING M. 1993: Species Evolution - the Role of Chromosome Change. Cambridge University Press, Cambridge, 336 pp.

Kirillova V.I. 1986: Chromosome numbers of the leafhoppers (Homoptera, Auchenorrhyncha) of the world fauna. I. Super- families Fulgoroidea, Cercopoidea and Cicadoidea. Entomol. Obozr. 65: 115-121 [in Russian, English abstr.].

KiRILLOVA V.I. 1989: The anatomy of the male reproductive system in Homoptera (Cicadinea, Delphacidae) and the use of its structure in the taxonomy of the family. Zool. Zh. 68: 143-148 [in Russian, English abstr.].

KuRoKawa H.A. 1953: A studies of the chromosomes in some species of the Cicadidae and the Cercopidae. Jap. J. Gen. 28: $1-5$.

Kuznetsova V.G. 1985: Phylogenetical analysis of the chromosome variability and karyosystematics of the leaf-hoppers of the family Dictyopharidae (Homoptera, Auchenorrhyncha). Entom. Obozr. 65: 88-106 [in Russian, English abstr.].

Kuznetsova V.G., Maryańska-Nadachowska A., Chung-Tu YANG \& O'BRIEN L. 1998: Karyotypes and structure of testes in cicads belonging to unsufficently known families of Fulgaroidea (Auchenorrhyncha, Homoptera). Folia Biol. (Kraków) 46: $23-40$.

Kuznetsova V.G., Maryańska-Nadachowska A. \& NokKala S. 2003: A new aproach to the Auchenorrhyncha (Hemiptera, Insecta) cytogenetics: Chromosomes of the meadow spittlebug Philaenus spumarius (L.) examined using various chromosome banding techniques. Fol. Biol. (Kraków) 51: 33-40.

Kuznetsova V.G., Grozeva S., Sewlal J-A.N. \& Nokkala S. 2007: Cytogenetic characterization of the Trinidad endemic Arachnocoris trinitatus Bergroth: the first data for the tribe Arachnocorini (Heteroptera: Cimicomorpha: Nabidae). Folia Biol. (Kraków) 55: 17-26.

Lanzone C. \& Souza M.J. 2006: Chromosome complement and meiosis in three species of the Neotropical bug genus Antiteuchus (Heteroptera, Pantetomodae, Discocephalidae). Genet. Mol. Biol. 29: 49-55.

Loukas M. \& Drosopoulos S. 1992: Population genetics of the spittlebug genus Philaenus (Homoptera: Cercopidae) in Greece. Biol. J. Linn. Soc. 46: 403-413.

Nechaeva G.A., Kuznetsova V.G. \& Nokkala S. 2004: New data on the karyotype of Pseudoccocus viburni (Sign.) (Homoptera, Coccnea). Entomol. Rev. 84: 393-400.

Nokkala S., Kuznetsova V.G., Maryańska-Nadachowska A. \& NoKKala C. 2004: Holocentric chromosomes in meiosis. I. Restriction of the number of chiasmata in bivalents. Chromosome Res. 12: 733-739.

PAPESCHI A.G. 1995: Correspondence between C-banding and Ag-NOR in the sex chromosomes of Belostoma oxyurum (Belostomatidae, Heteroptera). Cytologia 60: 291-295.

Remane R. \& Drosopoulos S. 2001: Philaenus tarifa nov. sp. an additional spittlebug from Southern Spain (Homoptera Cercopidae). Dt. Entomol. Z. 48: 277-279.

SCHWEIZER D. 1976: Reverse fluorescent chromosome banding with chromomycin and DAPI. Chromosoma 58: 307-324.

STEWARD A.J.A. \& LeEs D.R. 1996: The colour/pattern polymorphism of Philaenus spumarius (L.) (Homoptera: Cercopidae). Philos. Trans. R. Soc. Lond. (B) 351: 69-89.

SuMNER A.T. 1972: A simple technique for demonstrating centromeric heterochromatin. Exp. Cell. Res. 75: 304-306.

Received August 10, 2007; revised and accepted November 27, 2007 